\title{
Simulation analysis of equivalent circuit model of skin-electrode impedance for transcutaneous electrical stimulation
}

\author{
Joni Welman Simatupang ${ }^{1}$, Wilbert Wijaya ${ }^{2}$, David Tyler ${ }^{3}$, Clementine Mavridis ${ }^{4}$ \\ 1,2Electrical Engineering Study Program, Faculty of Engineering, President University, Cikarang, Indonesia \\ ${ }^{3,4}$ Fashion Institute, Manchester Metropolitan University (MMU), Manchester, United Kingdom
}

\section{Article Info \\ Article history: \\ Received Mar 13, 2021 \\ Revised May 20, 2021 \\ Accepted May 27, 2021}

\section{Keywords:}

Capacitive

Electrical stimulation

Impedance

Resistive

Transcutaneous

\begin{abstract}
For more than 50 years, transcutaneous electrical stimulation method has been used to cure the spinal cord injury, stroke or cerebral palsy. This method works by activating the excitable nerves, muscle fibers by electrical current stimulation through electrode to skin interface. Electrode to skin interface requires equivalent circuit to overcome the inability of measuring the skin resistivity directly. We have learned several previous models, which are from Lawler, Moineau and Keller and Kuhn. Unfortunately, Moineau model neglects the capacitance effect, while Lawler and Keller and Kuhn include capacitive and resistive nature of skin in their equivalent circuits. Both models consisted of only one parallel RC block. Therefore, this paper presents the simulation results of the proposed equivalent circuit model using two parallel $\mathrm{RC}$ circuits. Simulation of the proposed model is conducted in MATLAB 2015a and compared with two previous models using certain parameters. Results show that the proposed model obtained the impedance of $10.830 \mathrm{k} \Omega$ when it is simulated using $100 \mathrm{~Hz}$ frequency, for Lawler model the impedance is $5.340 \mathrm{k} \Omega$ and Keller and Kuhn model the impedance obtained is $6.490 \mathrm{k} \Omega$. The proposed model has the refined impedance compared with other models and is expected to deliver better electrical stimulation.
\end{abstract}

This is an open access article under the CC BY-SA license.

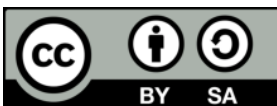

\section{Corresponding Author:}

Joni Welman Simatupang

Electrical Engineering Study Program

Faculty of Engineering, President University

Jababeka Education Park, Cikarang, Bekasi 17530, Indonesia

Email: joniwsmtp@ president.ac.id

\section{INTRODUCTION}

Wearable transcutaneous electrical stimulation (TES) or functional electrical stimulation (FES) is the method used to cure motoric injury due to stroke, spinal cord injury, or cerebral palsy, by activating excitable tissue from skin surface [1], [2]. This method has been applied in curing those diseases for $>50$ years [3]. TES or FES also works by activating the nerves and muscle fibers by stimulation using electrical current pulses through two electrodes and using inter-electrode distance (IED) which attached on the skin [4].

Electrical stimulation can be applied effectively for skin restoration, diagnosis and treatment purpose, by application of artificial tactile sensory feedback in prosthetic hand [5], [6]. Application of electrical stimulation requires highly reliable electrode, sustainable, and low-cost electrode material. Textile electrode fulfills these criteria as electrode material with price at $£ 0.16$ per electrode, which also provides high measurement accuracy and reusability [7]. Textile electrode is also providing flexibility, ventilation that can lead to almost no skin irritation for long-term usage, foldability and non-hydrogel characteristics. Textile electrodes is applicable for monitoring clinical physiological signals, such as electrocardiogram (ECG), electromyography (EMG), electroencephalogram (EEG), and so forth [8]. 
Textile electrode or e-textile technology has been around for almost a decade. E-textile permits the injection of electrical components, such as, LED, electrodes, to lay on the fabric for it to bring additional value to fabric [9]. The e-textile will be used as for future development of this research as wearable TES device, for this stage the main concern is designing the skin model for it to interface properly with human skin. The skin model design is made based on the skin two main characteristics, such as, resistive and capacitive, applying parallel RC circuit is the way to model after these characteristics. Resistivity of skin cannot be measured directly. Therefore, equivalent network model is used to obtain the skin resistance [2]. On the other hand, skin capacitance is measurable and frequency dependent, which can be measured with a device called Corneometer CM 825 for measurement using mean frequency of $1 \mathrm{MHz}$ or Corneometer CM 820 for low frequency measurement $(45-75 \mathrm{KHz})$ [10], [11]. The capacitance effect is useful to detect the hydration state of the skin and for biometric purpose [10]-[12]. Besides knowing the skin nature for stimulation purpose, current density in this stimulation plays a great role on the stimulation efficiency and comfort, due to the skin and electrodes in-homogeneities. High current density, which localized on the skin surface may cause discomfort and the worst case is skin burns [2].

Stimulation comfort on TES device can be improved by varying the waveform and pulse width [1], [5]. On previous research conducted by Baker et al., they have tried the stimulation using six different types of waveforms such as, asymmetric balanced biphasic square waveform, symmetric biphasic square waveform, monophasic paired spike, modulated sine wave, medium sine wave and medium square wave. Results of their experiment shows that asymmetric biphasic waveform provides better comfort and does not cause any interference or stimulation on large muscle group [13], therefore it is recommended for clinical usage. Equivalent circuit models have been made to substitute the skin model interface to determine the skin impedance. In this research, we limit to three model as our literature basis. First model was made in 1959 by Lawler et al. using the Wheatstone bridge approach with having both capacitive and resistive components to represents the skin nature. This research shows that impedance is inversely proportional to frequency [14]. Second model made by Keller \& Kuhn after the skin equivalent circuit from the skin layers [2]. Third model was made by Moineau et al., but excluded capacitance effect in their equivalent circuit model [3]. Those approaches yield different results in which, for Moineau et al. design it simply shows no changes in resistance, whereas on Lawler et al. and Keller \& Kuhn approach with including both resistive and capacitive nature of skin shows changes on the impedance with respect to frequency. Different equivalent circuit model has their own specialties, and also the waveform used in the stimulation may differ according to each purpose.

Electrode-skin interface is also playing an important role in electrical stimulation quality because higher impedance electrode will likely having low signal to noise $(\mathrm{S} / \mathrm{N})$ ratio, low signal amplitude and poor biological signal quality [15]. But on the other hand, higher electrode impedance may reduce discomfort in stimulation [1]. Increasing the resistivity in the electrode can be done by adding resistive gel, which act as impedance mismatch depletor. Size of electrode is affecting the current density uniformity, by applying electrode with larger contact area $\left(70-120 \mathrm{~cm}^{2}\right)$ may results in high current density and uniform current density, which results from the polarizable electrode characteristics, the displacement current [16]. This research is an extension work of David Tyler and Clementine Mavridis, from Manchester Metropolitan University (MMU), which our concern is in designing the more refined equivalent circuit model [17]. In this paper, the authors propose to simulate the model of Wheatstone bridge, which consists of two identical RC circuit. The RC circuit represents the resistive and capacitive nature of skin. The model will be simulated in MATLAB 2015a, with variation in resistance, capacitance and frequency. Wheatstone bridge approach is considered to be better at approximating the skin impedance, while on the other hand finding the perfect balance impedance in Wheatstone bridge is also more difficult.

\section{HUMAN SKIN}

Human skin is the largest and heaviest organ in the body which contribute 8-10\% of human body mass and covers about $2 \mathrm{~m}^{2}$ of our body's total area. Human skin also serves as boundaries between body and the environment such that, the vital bodily may perform under controlled physiological condition [18]-[20]. Ultraviolet (UV) light, chemicals, pathogens and mechanical injury are blocked by skin in first place [21]. Storing water and fats is also part of human skin's function and also serves as immunity to disease [22]. The main structure of human skin are epidermis, dermis and hypodermis [18]. Epidermis consist of several layers, such as, stratum corneum, stratum lucidum, stratum granulosum, stratum spinosum, stratum basale [21]. Dermis is the inner layer of supportive layer of skin, consist of vast amount of collagen and rich in blood supplies [22]. Hypodermis is the innermost layer of skin, which made up of subcutaneous fat [18].

Stratum corneum is the outermost of epidermis layer, which is the top layer of epidermis and human skin as a whole. Stratum corneum is made up of dead cell, or keratinized cell [4], [21], [22]. It is also one of the thinnest layers which has 15-20 $\mu$ m thickness only [23], [24]. Stratum corneum dominates the electronic

Simulation analysis of equivalent circuit model of skin-electrode impedance ... (Joni Welman Simatupang) 
properties of skin when DC current applied under $10 \mathrm{KHz}$ frequency [4], [23]. Resistivity of Stratum corneum is the highest amongst skin layer at about $10^{5} \Omega \mathrm{cm}^{2}$, and conductivity of $0.03 \mu \mathrm{F} / \mathrm{cm}^{2}$ at small current application [4], [24]. Dermis is the second main layer of human skin with thickness of 1 to $4 \mathrm{~mm}$, consists of two layers, such as, papillary layer and reticular layer. Papillary layer is the upper layer of dermis, which serves as boundary to epidermis, loosely connected tissue, with large amount of nerve fibers, capillaries, water and cells. Reticular layer is the bottom part of dermis, which has denser and thicker network compare to papillary layer, houses fewer nerve fibers and capillaries [19].

\subsection{Skin circuit model}

Skin circuit model is required for electrical stimulation due to the immeasurable impedance of it [2]. Making a skin circuit model is the way to simplify our analysis. Skin circuit is modeled by an equivalent circuit, consist of RC circuit. Figure 1 shows the equivalent circuit model of skin [23].

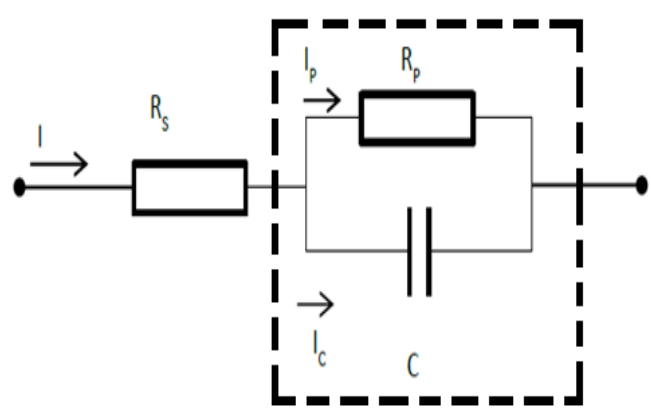

Figure 1. Skin equivalent circuit model

This skin model is the common way to represent the dielectric properties of skin and skin general response. Analysis of signal input and output from body is simplified with the modelling of this circuit. This model is the most common used for measuring the changes in impedance. In addition, this model is considered to be adequate enough to characterize the skin response [23].

In this research the parallel RC circuit will be integrated with Wheatstone bridge circuit which was invented in 1833 by Samuel H. Christie, then improved and popularized by Sir Charles Wheatstone in 1843 [25], [26]. This circuit model is intended to figure out the unknown resistance, using the defined resistance in a bridge model circuit compare with the unknown resistance [26], [27]. This is suitable for measuring skin resistance for it cannot be measured directly. Small changes in resistance can be measured by Wheatstone bridge circuit [26]. Wheatstone bridge can be used for measuring capacitance, inductance, and impedance. Wheatstone bridge circuit is used for measuring the unknown parameter for DC source. In balance state, Wheatstone bridge circuit has $0 \mathrm{~V}$ voltage across the bridge, therefore, when small changes occur voltage difference exists [25].

\subsection{The previous and proposed models}

In this part, we will be discussing the skin model made by Lawler et al., and Keller \& Kuhn, and Moineau et al. First, Lawler et al. proposed the model in 1959 Figure 2, stated that skin has both resistive and capacitive nature. They also concluded that impedance of skin will be the same as equivalent circuit in balance condition. Therefore, Lawler et al. come up with the model based of Wheatstone bridge for the skin to have zero voltage in balance state and construct the skin model by using parallel RC circuit, for it to have equal impedance with the skin. This skin model is connected to one of the bridge legs and opposing the electrode.

Second, Keller \& Kuhn [2] proposed their comprehensive skin model with consist of the matrix and appendageal pathway. The pathways are modeled with resistor and capacitors. In their research, Keller and Kuhn obtained the skin resistivity by tuning the equivalent circuit for yielding the measured current-voltage response. Keller and Kuhn's simple lumped skin model is shown in Figure 3. Impedance formula of Lawler et al. model is shown in (1) 


$$
Z=\frac{R \cdot\left(\frac{1}{2 \pi f C}\right)}{\sqrt{R^{2}+\left(\frac{1}{2 \pi f C}\right)^{2}}}
$$

The impedance formula of the simple lumped skin model is shown in (2)

$$
Z=\frac{R \cdot\left(\frac{1}{2 \pi f C}\right)}{\sqrt{R^{2}+\left(\frac{1}{2 \pi f C}\right)^{2}}}+R_{b}+R_{c}
$$

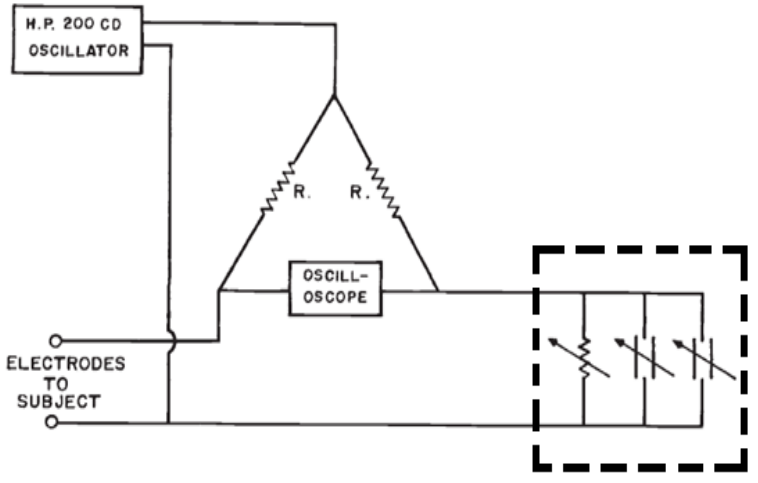

Figure 2. Lawler et al. skin model

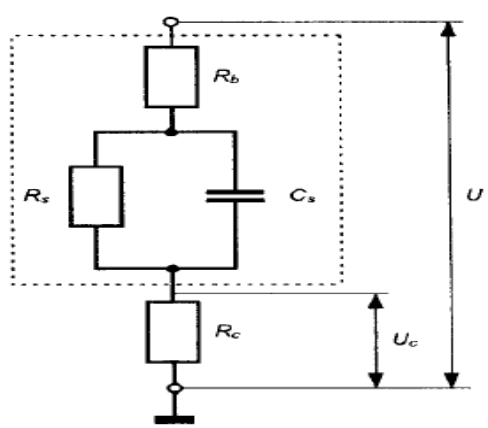

Figure 3. Simple lumped skin model

Third, Moineau et al. come up with the model only consisting resistive nature of skin, therefore the impedance of the circuit is by using voltage on each node of the circuit (can be solved by using Ohm's law). The results yield without taking account any capacitance effect. In this way we obtained a distorted skin impedance value that can't be comparable with the one of the Wheatstone bridge models [3], [9].

The proposed model is designed based on Wheatstone bridge as used in Lawler et al. model as shown in Figure 4. The impedance of the model is determined by the block marked with black dashed line. The impedance formula will be elaborated starting from (3) to (6), and the final formula of the impedance will be shown in (6).

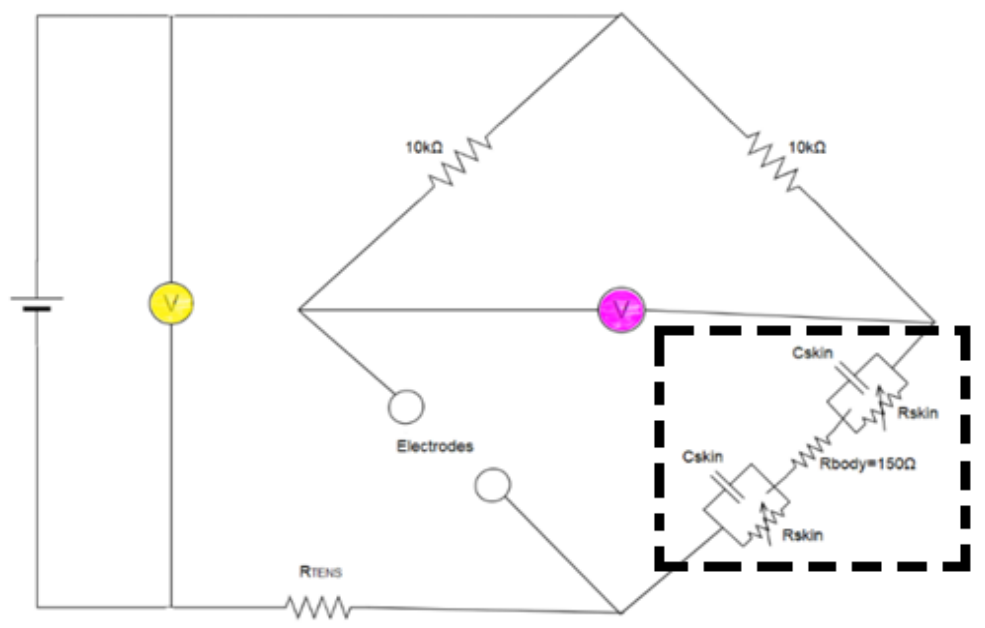

Figure 4. The proposed equivalent circuit for simulation is blocked with the black dashed line 


$$
\begin{aligned}
& Z=\frac{R_{\text {skin }} \cdot X_{c}}{\sqrt{R_{\text {skin }}^{2}+X_{c}^{2}}} \\
& X_{C}=\frac{1}{2 \pi f C_{\text {skin }}} \\
& Z=\frac{R_{\text {skin }} \cdot\left(\frac{1}{2 \pi f C_{\text {skin }}}\right)}{\sqrt{{R_{\text {skin }}}^{2}+\left(\frac{1}{2 \pi f C_{\text {skin }}}\right)^{2}}}
\end{aligned}
$$

Therefore, the impedance for this circuit is:

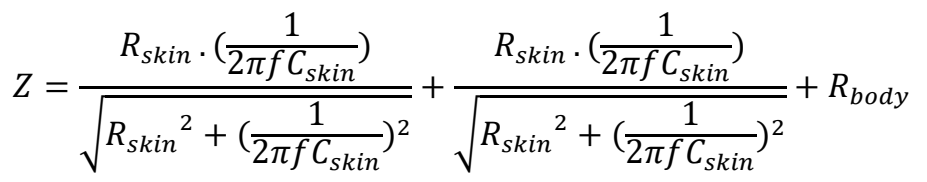

Table 1 shows that models from Lawler et al. and Keller \& Kuhn include capacitance and resistance nature of skin, in which the equivalent circuit can be solved for the impedance and the impedance is affected by changes in frequency. Impedance formula of both Lawler et al. and Keller \& Kuhn models are shown in (1) to (6) and from the formula can be inferred that impedance is inversely proportional to frequency. Moineau's is the least model among those previous models and will be neglected later in our simulations.

Table 1. Performance characteristic between three previous models and the proposed model

\begin{tabular}{ccccc}
\hline Model/Parameter & Impedance & Capacitance & Resistance & Frequency \\
\hline Lawler et al. & $\checkmark$ & $\checkmark$ & $\checkmark$ & $\checkmark$ \\
Moineau et al. & $\boldsymbol{x}$ & $\boldsymbol{x}$ & $\checkmark$ & $\checkmark$ \\
Keller \& Kuhn & $\checkmark$ & $\checkmark$ & $\checkmark$ & $\checkmark$ \\
Proposed Model & $\checkmark$ & $\checkmark$ & $\checkmark$ & $\checkmark$ \\
\hline
\end{tabular}

\section{SIMULATION RESULTS AND ANALYSIS}

In this section, the results of simulation from the proposed model, Lawler et al., and Keller \& Kuhn models will be elaborated. Table 2 shows the parameters used to simulate the proposed model, Lawler et al. and Keller \& Kuhn model, for it to be further analyzed. The frequency is chosen variably from 10 to $150 \mathrm{~Hz}$. In the first, impedance vs. frequency is simulated by varying the frequency with ranging from $10 \mathrm{Ho} 150 \mathrm{~Hz}$. The results of simulation are shown in Figures 5-7. In Figure 5, we can see that the highest impedance of the proposed model is $19.990 \mathrm{~K} \Omega$ and the lowest impedance of $7.911 \mathrm{~K} \Omega$, Lawler et al. model showing highest impedance of $9.877 \mathrm{~K} \Omega$ and lowest impedance of $3.881 \mathrm{~K} \Omega$ and Keller and Kuhn Model shows highest impedance of $11.027 \mathrm{~K} \Omega$ and lowest impedance of $5.031 \mathrm{~K} \Omega$. From these results, it can be concluded that the impedance is inversely proportional to frequency.

Table 2. Simulation parameters

\begin{tabular}{ccccccc}
\hline Models & $\mathrm{R}_{\text {skin }}(\mathrm{K} \Omega)$ (fixed) & $\mathrm{R}_{\text {skin }}(\mathrm{K} \Omega)($ variable $)$ & $\mathrm{R}_{\text {body }}(\Omega)$ & $\mathrm{R}_{\mathrm{b}}(\Omega)$ & $\mathrm{R}_{\mathrm{c}}(\mathrm{K} \Omega)$ & $\mathrm{C}_{\text {skin }}(\mathrm{nF})(\mathrm{variable})$ \\
\hline Proposed Model & 10 & $10-100$ & 150 & - & - & $83-251$ \\
Lawler et al. & 10 & $10-100$ & - & - & - & $83-251$ \\
Keller and Kuhn & 10 & $10-100$ & - & 150 & 1 & $83-251$ \\
\hline
\end{tabular}

Then, impedance vs. resistance is simulated by varying resistance ranging from $10 \mathrm{~K} \Omega$ to $100 \mathrm{~K} \Omega$, and frequency of $100 \mathrm{~Hz}$. Proposed model results in the increment of $1.365 \mathrm{~K} \Omega$ from 10 to $20 \mathrm{~K} \Omega$, Lawler et al. and Keller \& Kuhn model showing increment of $0.683 \mathrm{~K} \Omega$ from 10 to $20 \mathrm{~K} \Omega$, from $30 \mathrm{~K} \Omega$ to $100 \mathrm{~K} \Omega$ just small increment occur. The results of simulation are shown in Figure 6. Finally, impedance vs. capacitance of proposed model, Lawler et al. and Keller \& Kuhn are simulated by varying the capacitance value ranging from 83 to $251 \mathrm{nF}$. The proposed model yields the highest impedance of $17.883 \mathrm{~K} \Omega$ and lowest impedance of $10.860 \mathrm{~K} \Omega$, Lawler et al. model highest impedance is $8.867 \mathrm{~K} \Omega$ and lowest impedance of $5.355 \mathrm{~K} \Omega$, Keller 
\& Kuhn model highest impedance is $10.017 \mathrm{~K} \Omega$ and lowest impedance of $6.505 \mathrm{~K} \Omega$. The results are shown in Figure 7. From the results, we can conclude that capacitance is inversely proportional to impedance.

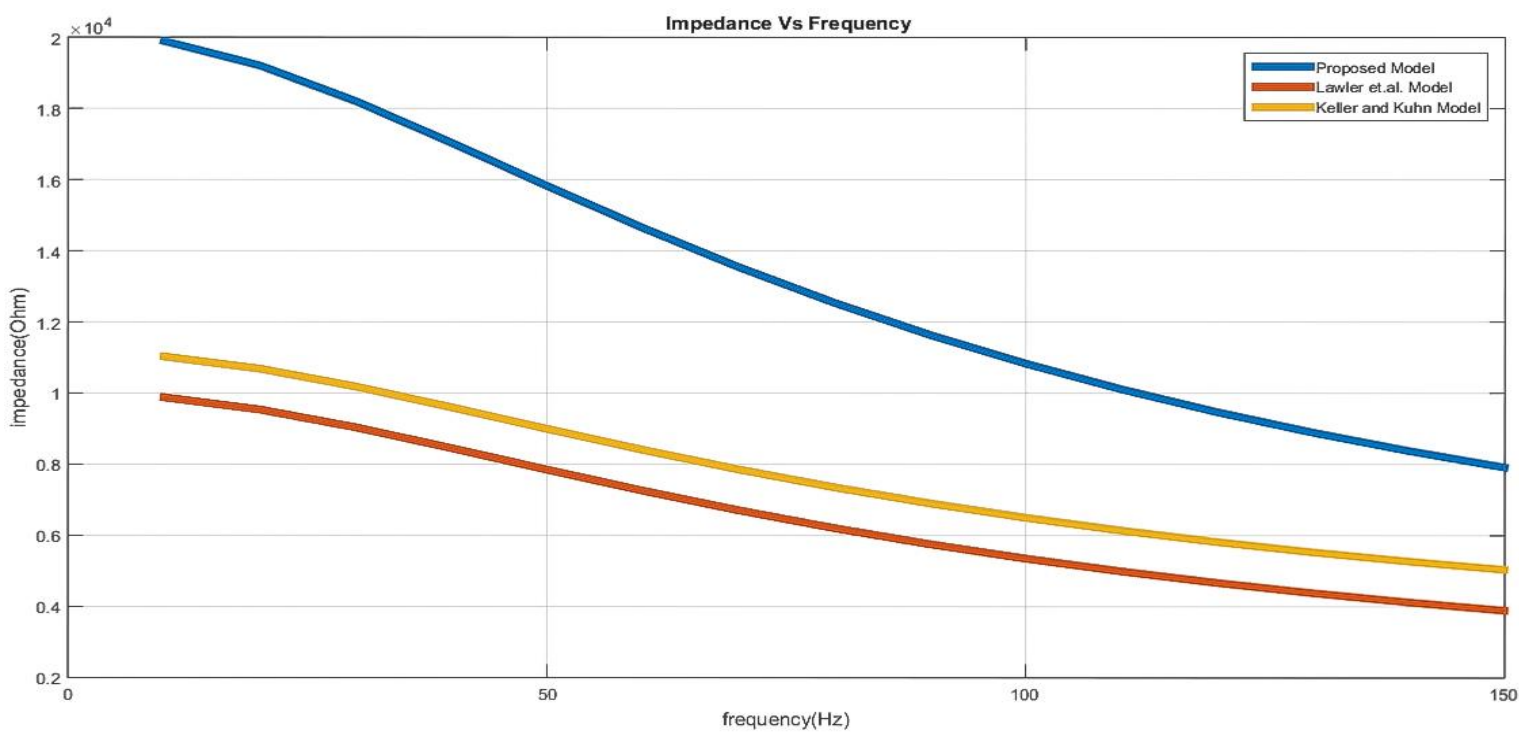

Figure 5. Impedance vs. frequency of proposed model, Lawler et al. and Keller and Kuhn from 10-150 Hz

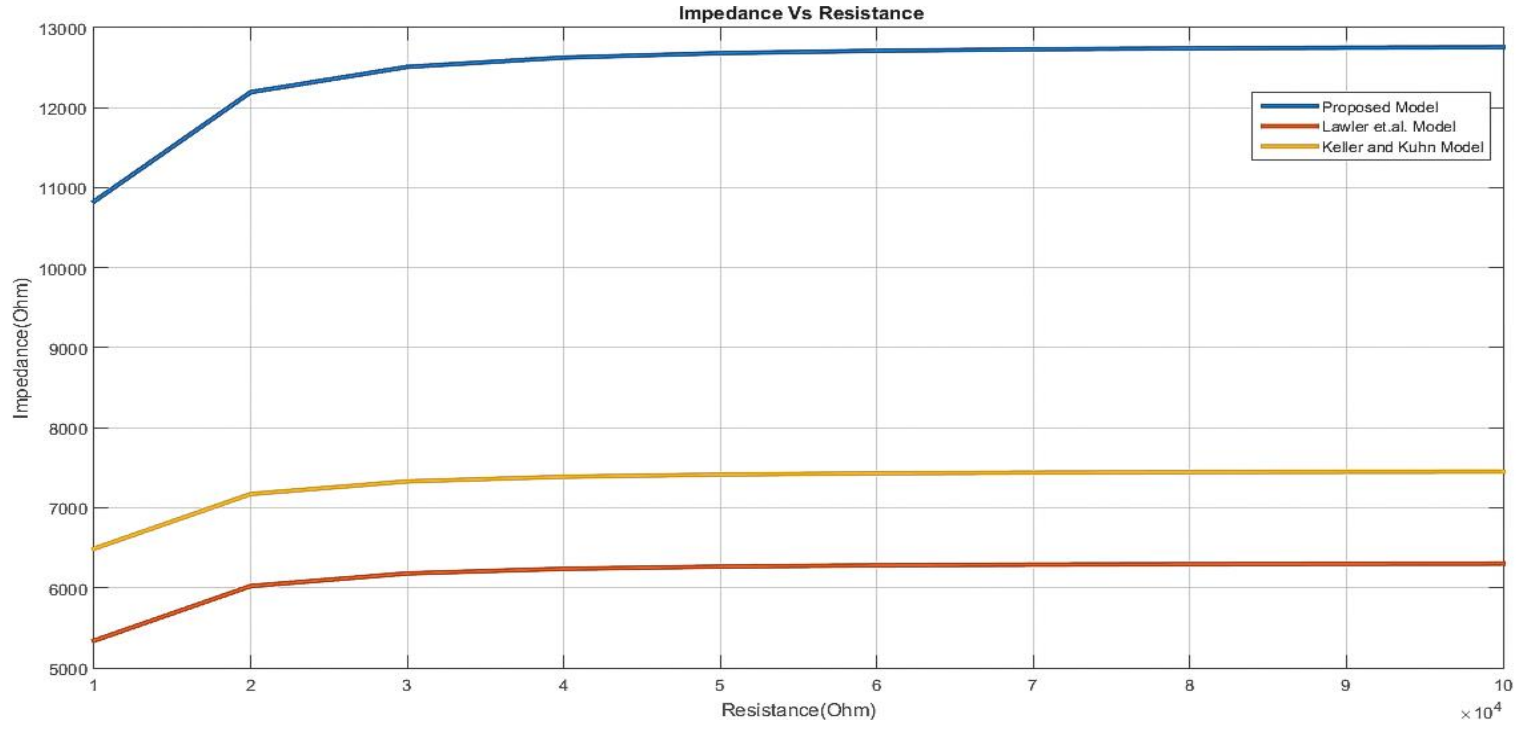

Figure 6. Impedance vs. resistance of proposed model, Lawler et al. and Keller and Kuhn from 10-100 K

Then, impedance vs. resistance is simulated by varying resistance ranging from $10 \mathrm{~K} \Omega$ to $100 \mathrm{~K} \Omega$, and frequency of $100 \mathrm{~Hz}$. Proposed model results in the increment of $1.365 \mathrm{~K} \Omega$ from 10 to $20 \mathrm{~K} \Omega$, Lawler et $a l$. and Keller \& Kuhn model showing increment of $0.683 \mathrm{~K} \Omega$ from 10 to $20 \mathrm{~K} \Omega$, from $30 \mathrm{~K} \Omega$ to $100 \mathrm{~K} \Omega$ just small increment occur. The results of simulation are shown in Figure 6. Finally, impedance vs. capacitance of proposed model, Lawler et al. and Keller \& Kuhn are simulated by varying the capacitance value ranging from 83 to $251 \mathrm{nF}$. The proposed model yields the highest impedance of $17.883 \mathrm{~K} \Omega$ and lowest impedance of $10.860 \mathrm{~K} \Omega$, Lawler et al. model highest impedance is $8.867 \mathrm{~K} \Omega$ and lowest impedance of $5.355 \mathrm{~K} \Omega$, Keller \& Kuhn model highest impedance is $10.017 \mathrm{~K} \Omega$ and lowest impedance of $6.505 \mathrm{~K} \Omega$. The results are shown in Figure 7. From the results, we can conclude that capacitance is inversely proportional to impedance. 


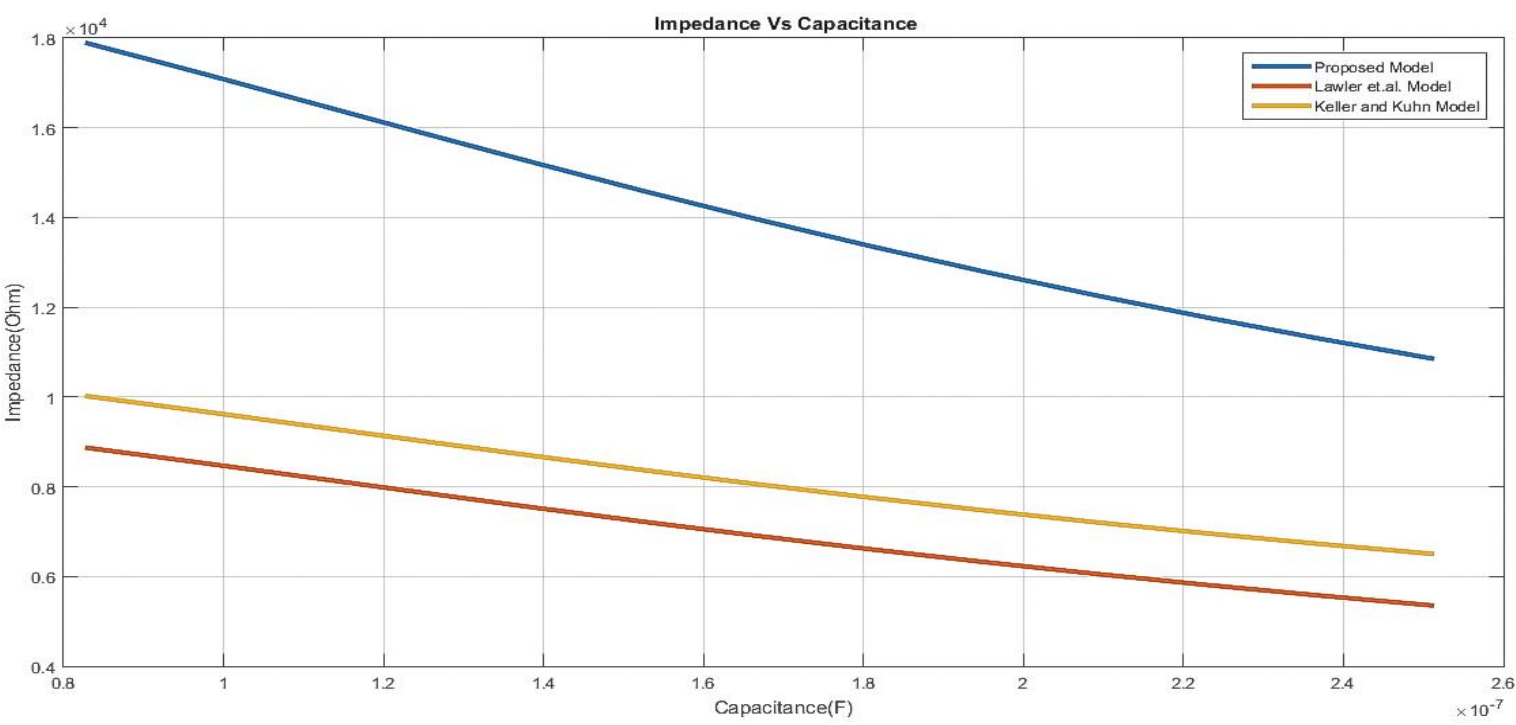

Figure 7. Impedance vs. capacitance of proposed model, Lawler et al. and Keller and Kuhn from 83-251 nF

In this research, the frequency is affecting the accuracy of the measurement on high frequency which can cause error due to the changing current distribution beneath electrodes surface and low frequency error due to the impedance imbalance, causing $\mathrm{V} \neq 0$ [27]. Low frequency impedance can be used for indicating skin burn [28]. In addition, at low frequency impedance reflects the resistive properties of extracellular environment. Higher frequency impedance is reflecting both resistive and capacitive properties of intra and extra-cellular environments. The overall impedance may state the clinical state of human skin [29]. The overall impedance of human tissue is in $\mathrm{K} \Omega$ range, measurement made on human impedance using high accuracy bioimpedance measurement system by this following set up, frequency ranging from $24 \mathrm{KHz}$ to $391 \mathrm{KHz}$, excitation current of $10 \mu \mathrm{A}$, and resulting $37 \mathrm{~K} \Omega$ of impedance [30].

From the results comparison of proposed model, Lawler et al. and Keller \& Kuhn model, by variation in frequency, resistance and capacitance, all of the impedance results showing that the proposed model shows the highest impedance, followed by Keller \& Kuhn, then Lawler et al. The proposed model showed a refinement in terms of impedance. Having this quality, proposed model is expected to deliver better comfort stimulation.

\section{CONCLUSION AND FUTURE WORKS}

Results yield from our simulations show that the proposed model has the highest impedance, followed by Keller \& Kuhn, then Lawler et al. models. Therefore, the proposed model is considered to be the refinement product compared to other models and expected to deliver better comfort in electrical stimulation. For future works, adding low pass filter might be good to give a better signal to noise $(\mathrm{S} / \mathrm{N})$ ratio. Also, by modeling the equivalent circuit using Thevenin scheme, with including the parallel RC circuit and resistance.

\section{ACKNOWLEDGEMENTS}

Thank to Research Institute and Community Service of President University for supporting our work.

\section{REFERENCES}

[1] N. Sha, L. Kenney, B. Heller, A. Barker, D. Howard, "A Finite Element Model to Identify Electrode Influence on Current Distribuution in the Skin," Artificial Organs, vol. 32, pp. 639-43, 2008, doi: https://doi.org/10.1111/j.15251594.2008.00615.x.

[2] T. Keller, A. Kuhn, "Electrodes for transcutaneous (surface) Electrical Stimulation," Journal of Automatic Control, University of Belgrade, vol. 18, no. 2, pp. 35-45, 2008, doi: https://doi.org/10.2298/JAC0802035K.

[3] B. Moineau et al., "Garments for Functional Electrical Stimulation: Design and Proofs of Concept," Journal of Rehabilitation and Assistive Technologies Engineering, vol. 6, pp.1-15, 2019, doi: https://doi.org/10.1177/2055668319854340. 
[4] M. Sacilotto et al., "A Simpler and Shorter Neuromuscular Electrical Stimulation Protocol Improves Functional Status and Modulates Inflammatory Profile in Patients with End-Stage Congestive Heart Failure," International Journal of Cardiovascular Sciences, vol. 30, no. 6, pp. 484-494, 2017, doi: https://doi.org/10.5935/23594802.20170064.

[5] J. L. V. Luna, M. Krenn, J. A. C. Ramirez, W. Mayr, "Dynamic Impedance Model of the Skin-Electrode Interface for Transcutaneous Electrical Stimulation," Plos One, pp. 1-15, 2015, doi: https://doi.org/10.1371/journal.pone.0125609.

[6] K. Zhu, L. Li, X. Wei, X. Sui, "A 3D Computational odel of Transcutaneous Electrical Nerve Stimulation for Estimating A $\beta$ Tactile Nerve Fiber Excitability," Frontiers in Neuroscience, vol. 11, no. 250, pp. 1-15, 2017, doi: https://doi.org/10.3389/fnins.2017.00250.

[7] S. Pitou, F. Wu, A. Shafti, B. Michael, R. Stopforth, M. Howard, "Embroidered Electrodes for Control of Affordable Myoelectric Prostheses," 2018 IEEE International Conference on Robotics and Automation (ICRA), King's College London 2018, pp. 1812-1817, doi: 10.1109/ICRA.2018.8461066.

[8] H. Zhou, Y. Lu, W. Chen, Z. Wu, H. K. L. Zou, L. Guanglin, "Stimulating the Comfort of Textile Electrodes in Wearable Neuromuscular Electrical Stimulation," Sensors, vol. 15, no. 7, pp. 17241-17257, 2015, doi: https://doi.org/10.3390/s150717241.

[9] C. Mavridis, "Internship report Engineering Assistant - Wearable technology," Manchester Metropolitan University (MMU), Manchester, United Kingdom, 2019.

[10] M. Qassem, P. Kyriacou, "Review of Modern Techniques for the Assessment of Skin Hydration," Cosmetics, vol. 6, no. 19, pp. 1-28, 2019, doi: https://doi.org/10.3390/cosmetics6010019.

[11] V. Zuang, C. Rona, F. Distante, E. Berardesca, "The Use of a Capacitance Device to Evaluate The Hydration of Human Skin,” Journal Application Cosmetol, vol. 15, pp. 95-102, 1997.

[12] C. Bontozoglou, P. Xiao, "Applications of Capacitive Imaging in Human Skin Texture and Hair Analysis," Applied Sciences, vol. 10, no. 256, pp. 1-13, 2020, doi: https://doi.org/10.3390/app10010256.

[13] L. L. Baker, B. R. Bowman, D. R. McNeal, "Effects of Waveform on Comfort During Neuromuscular Electrical Stimulation," Clinical Orthopaedics and Related Research, 1987.

[14] J.C. Lawler, M. J. Davis, E. C. Griffith, "Electrical Characteristics of The Skin: The Impedance of The Surface Sheath and Deep Tissues," Journal Investigative Dermatology, vol. 34, no. 5, pp. 301-308, 1960.

[15] A. Albulbul, "Evaluating Major Electrode Types for Idle Biological Signal Measurements for Modern Medical Technology," Bioengineering, vol. 3, no. 20, pp. 1-10, 2016, doi: https://doi.org/10.3390/bioengineering3030020.

[16] J. Kim, G. Yoon, "Study on Reusable Electrodes for Personal Electrocardiography," Journal of Sensor Science and Technology, vol. 27, no. 5, pp. 340-344, 2018, doi: https://doi.org/10.5369/JSST.2018.27.5.340.

[17] D. Tyler, C. Mavridis, "Wearable FES Electrodes," in Proceedings MDPI, Basel, Switzerland, 2019, vo. 32, no. 13, pp. 1-4, doi: 10.3390/proceedings2019032013.

[18] S. Zsikó, E. Csányi, A. Kovács, M. B. Szucs, A. Gácsi, S. Berkó, "Methods to Evaluate Skin Penetration In Vitro," Scientia Pharmaceutica, vol. 87, no. 19, pp. 1-21, 2019, doi:10.3390/scipharm87030019.

[19] J. M. Abdo, N. A. Sopko, S. M. Milner, "The applied anatomy of human skin: A model for regeneration," Wound Medicine, vol. 28, 2020, doi: https://doi.org/10.1016/j.wndm.2020.100179.

[20] F. Lu, C. Wang, R. Zhao, L. Du, Z. Fang, X. Guo, Z. Zhao, "Review of Stratum Corneum Impedance Measurement in Non-Invasive Penetration Application," Biosensors, vol. 8, no. 31, pp. 1-20, 2018, doi:10.3390/bios8020031.

[21] H. Yousef, S. Sharma, “Anatomy, Skin (Integument), Epidermis,” National Institutes of Health, 2017.

[22] C. Gorzelanny et al., "Skin Barriers in Dermal Drug Delivery: Which Barriers Have to Be Overcome and How Can We Measure Them?," Pharmaceutics, vol. 12, no. 684, pp. 1-31, 2020, doi: 10.3390/pharmaceutics12070684.

[23] L. Davis, "A Cellular Model of the Electrical Characteristics of Skin,” University of Southampton, 2018.

[24] Y. A. Chizmadzhev, A. V. Indenbom, P. I. Kuzmin, S. V. Galichenko, J. C. Weaver, Russell O. Potts, "Electrical Properties of Skin at Moderate Voltages: Contribution of Appendageal Macropores," Biophysical Journal, vol. 74, no. 2, pp. 843-856, 1998, doi: https://doi.org/10.1016/S0006-3495(98)74008.

[25] T.-Y. Wu, et al., "CircuitSense: Automatic Sensing of Physical Circuits and Generation of Virtual Circuits to Support Software Tools," UIST '17: Proceedings of the 30th Annual ACM Symposium on User Interface Software and Technology, 2017, pp. 311-319, https://doi.org/10.1145/3126594.3126634.

[26] K. Hoffmann, "Applying The Wheatstone Bridge Circuit," [Online]. Available: http://eln.teilam.gr/sites/default/files/Wheatstone\%20bridge.pdf. 2021.

[27] O. Ibrahim, S. M. Hassan, A. Abdulkarim, M. F. Akorede, S. Amuda, Sulayman A.Y, "Design of Wheatstone Bridge Based Thermistor Signal Conditioning Circuit for Temperature Measurement," Journal of Engineering Science and Technology Review, vol. 12, no. 1, pp. 12-17, 2019.

[28] L. Yang et al., "The Frequency Spectral Properties of Electrode-Skin Contact Impedance on Human Head and Its Frequency-Dependent Effects on Frequency-Difference EIT in Stroke Detection from $10 \mathrm{~Hz}$ to $1 \mathrm{MHz}$," Plos One, pp. 1-21, 2017, doi: https://doi.org/10.1371/journal.pone.0170563.

[29] K. A. Sillay, et al., "Long-Term Surface Electrode Impedance Recordings Associated with Gliosis for a ClosedLoop Neurostimulation Device," Annals of Neurosciences, vol. 25, pp. 289-298, 2018, doi: https://doi.org/10.1159/000481805

[30] U. Birgersson, "Electrical Impedance of Human Skin and Tissue Alterations: Mathematical Modeling and Measurements," Karolinska Institutet, Campus Huddinge, 2012. 